\title{
Tuberculosis y SIDA, una confusa combinación
}

La tuberculosis puede considerarse como una de las enfermedades que más vidas ha cobrado a lo largo de la historia de la humanidad, pues es la responsable, en los últimos 200 años, de más de un millón de muertes. A pesar de todos los conocimientos disponibles en la actualidad, no se ha logrado su derrota, de ahí que continúe siendo la enfermedad infecciosa más importante de nuestros tiempos e infortunadamente el reflejo de las enormes diferencias socioeconómicas de la sociedad moderna (1-5).

A finales del siglo anterior fuimos testigos del resurgimiento de la tuberculosis en países en vías de desarrollo y en muchos desarrollados, en parte por la puesta en escena de la pandemia de SIDA, lo cual además de aumentar el número de casos, produjo la reaparición de otros más difíciles de diagnosticar, no solo por su variación en la presentación clínica, sino por la misma localización de la enfermedad, como se manifiesta en el estudio realizado en el Hospital Santa Clara que se publica en este número de la Revista (6). Es así como el SIDA ha hecho que la tuberculosis recobre los adjetivos de gran simuladora.

Es bien conocido que a mayor grado de inmunosupresión del paciente con SIDA, mayor probabilidad de aparición de presentaciones atípicas de la tuberculosis, lo cual incluye formas extrapulmonares e implica, en la mayor parte de los casos, más dificultades en el diagnóstico y el requerimiento de más tiempo y procedimientos invasivos, incluyendo análisis bacteriológicos de toda clase de muestras y biopsias de tejidos (1-2).

Estas formas extrapulmonares se pueden manifestar solas o asociadas a formas pulmonares de la tuberculosis en el paciente con SIDA, siendo frecuentes los compromisos de serosas como pleura, pericardio y peritoneo, con extensos compromisos ganglionares y siembras miliares en diferentes órganos (1-2).

Por otra parte y aumentando un poco más la magnitud del problema, una presentación clínica e histológica similar puede aparecer en pacientes con enfermedad por micobacterias no tuberculosas, que de otro lado son frecuentes en el paciente con SIDA, en especial cuando hay una inmunosupresión severa, haciendo que ya no solo sean indispensables para un adecuado diagnóstico las técnicas microbiológicas convencionales, sino el uso de nuevos métodos basados en amplificación genética que permiten hacer tipificación de la micobacteria, y que por supuesto requieren mayor infraestructura (3-5).

Es claro que la asociación de tuberculosis con SIDA empeora considerablemente el pronóstico de ambas enfermedades, y hasta hace poco más de una década cerca de la mitad de los pacientes con esta combinación morían antes de completar el tratamiento de la tuberculosis. Por fortuna esto viene cambiando, en especial con la introducción temprana de antirretrovirales, específicamente en aquellos pacientes con bajos conteos de linfocitos CD4, como lo demuestran estudios publicados en el año 2011 en el New England Journal of Medicine, por los investigadores del equipo CAMELIA y por los grupos de los doctores Salim y Havlir, en los cuales se comprobó que hay beneficio con disminución en la mortalidad, hecho que cambió la manera de tratar este tipo de pacientes $(7,8)$.

Podría resumirse que los principales problemas a los cuales se enfrenta el médico en la asociación tuberculosis-SIDA son la mayor dificultad en el diagnóstico, los cambios en el tratamiento y más reacciones adversas a medicamentos, lo cual se refleja en mayor tasa de abandonos y mayor número de interacciones medicamentosas y reacciones sistémicas como el síndrome de reconstitución inmunológica.

Puede decirse además, que la aparición del SIDA ha convertido la eliminación de la tuberculosis en un objetivo un poco más lejano, pero al mismo tiempo en un incentivo para la comunidad científica, las autoridades 
sanitarias y la sociedad en general, pues conlleva ingeniar nuevos adelantos y políticas que logren sacar victoriosa a la humanidad de esta gran calamidad.

El estudio realizado en el Hospital Santa Clara, reafirma la importancia de buscar la presencia de coinfección de tuberculosis y VIH, ya que en este contexto la presentación clínica de las dos enfermedades, y específicamente para el caso de la tuberculosis, el diagnóstico requiere un alto grado de sospecha y búsqueda en órganos diferentes al pulmón, lo cual por supuesto implica mayor uso de recursos diagnósticos y nuevas tecnologías, que aún en nuestro medio no están disponibles de forma masiva $(4,5)$.

Por otro lado, este estudio debe servir como incentivo para quienes trabajan en los programas de tuberculosis con miras a llevar un registro adecuado de los pacientes, ya que no solo es una buena fuente de investigación, sino que además ayuda a afrontar los retos que esta cambiante y fascinante patología impone en labor diaria, con base en un mejor conocimiento de la población propia.

\section{BIBLIOGRAFÍA}

1. Swaminathan S, Padmapriyadarsini C, Narendran G. HIV-associated tuberculosis: Clinical Update. Clin Infect Dis. $2010 ; 50: 1377-86$.

2. Sterling TR, Pham PA, Chaisson RE. HIV infection -related tuberculosis: Clinical manifestations and treatment. Clin Infect Dis. 2010; 50: S223-S30.

3. Gupta R K, Lawn S D, Bekker L-G, Caldwell J, Kaplan R, Wood R. Impact of human immunodeficiency virus and CD4 count on tuberculosis diagnosis: analysis of city-wide data from Cape Town, South Africa. Int J Tuberc Lung Dis. 2013; 17: 1014-1022.

4. Cain K, McCarthy K, Heilig M, Monkongdee P, Tasaneeyapan T, et al. An algorithm for tuberculosis screening and diagnosis in people with HIV. N Engl J Med. 2010; 362: 707-716.

5. Zumla A, Raviglione M, Hafner R, von Reyn F. Tuberculosis. N Engl J Med 2013; 368: 745-55.

6. Fenner L, Gagneux S, Janssens J, Fehr J, Cavassini M, et al Tuberculosis in HIV-Negative and HIV-Infected patients in a low-incidence country: Clinical Characteristics and treatment outcomes. PlosOne 2012; 7: e34186.

7. Salim S, Karim A, Naidoo K, Grobler A, Padayatchi N, Baxter C, et al. Integration of antiretroviral therapy with tuberculosis treatment. N Engl J Med. 2011; 365: 1492-501.

8. Blanc F, Sok T, Laureillard D, Borand L, Pharm D, Rekacewicz C, et al. Earlier versus later start of antiretroviral therapy in HIV-Infected adults with tuberculosis. N Engl J Med. 2011; 365: 1471-81.

Juan Carlos Rojas, MD. Internista, Neumólogo. Hospital Universitario San Ignacio. Pontificia Universidad Javeriana. Bogotá, Colombia. Correo electrónico: jrojaspuentes@gmail.com 